\title{
Response of molars and non-molars to a strict supragingival control in periodontal patients
}

Patrícia Daniela Melchiors Angst ${ }^{(a)}$ Flávia Benetti Piccinin ${ }^{(a)}$

Rui Vicente Oppermann ${ }^{(a)}$

Rosemary Adriana Chiérici

Marcantonio ${ }^{(b)}$

Sabrina Carvalho Gomes ${ }^{(a)}$

(a) Department of Periodontology, Dental School, Univ Federal do Rio Grande do Sul - UFRGS, Porto Alegre, RS, Brazil.

(b) Department of Periodontology, Univ Estadual Paulista - Unesp, Araraquara, SP, Brazil.
Declaration of Interests: The authors certify that they have no commercial or associative interest that represents a conflict of interest in connection with the manuscript.

Corresponding Author: Sabrina Carvalho Gomes

E-mail: sabrinagomes.perio@gmail.com

Submitted: Aug 24, 2012

Accepted for publication: Nov 30, 2012

Last revision: Nov 30, 2012
Abstract: The posterior position in the arches is one of the factors that underlies the poor prognosis of molar teeth (M). It is speculated that $\mathrm{M}$ do not benefit from the oral hygiene routine as well as non-molars (NM) do. This study evaluated the response of $M$ and NM to supragingival control during a 6 -month period in 25 smokers $(\mathrm{S})$ and 25 never-smokers (NS) with moderate-to-severe periodontitis. One calibrated examiner assessed visible plaque (VPI) and gingival bleeding (GBI) indexes, periodontal probing depth (PPD), bleeding on probing (BOP), and clinical attachment loss (CAL) at days 0 (baseline), 30 and 180. At baseline, $\mathrm{M}$ showed significantly higher mean values of VPI $(\mathrm{p}=0.017)$ and PPD $(\mathrm{p}<0.001)$ compared with NM; CAL was also greater in $\mathrm{M}(\mathrm{p}<0.001)$ and was affected by smoking $(\mathrm{p}=0.007)$. The reductions obtained for periodontal indicators at day 180 showed similar responses between $\mathrm{M}$ and NM. For CAL, M (NS $0.57 \pm 0.50 ; S 0.67 \pm 0.64$ ) and NM (NS $0.38 \pm 0.23$; S $0.50 \pm 0.33)$ reached an almost significant difference $(\mathrm{p}=0.05)$. Smoking did not influence the response to treatment. Multilevel analysis revealed that, only for PDD reductions, the interaction between sites, teeth and patient was significant $(\mathrm{p}<0.001)$. It was concluded that $\mathrm{M}$ benefit from an adequate regimen of supragingival biofilm control; therefore, supragingival condition should be considered in the prognosis of molar teeth.

Descriptors: Tooth; Smoking; Periodontitis; Dental Scaling.

\section{Introduction}

Supragingival control has been considered a sine qua non condition to the establishment and maintenance of periodontal health. ${ }^{1,2}$ Although this issue is not recent, the evidence that reinforces that supragingival control influences the subgingival environment to a large extent, both from a clinical and a microbiological standpoint, is recent. ${ }^{3-5}$ Thus, considering this relationship, no subgingival therapy is successful when an adequate control of supragingival biofilm is not ensured. However, an adequate supragingival plaque control depends on various factors, including the recognized difficulty to obtaining patient compliance regarding to the daily routine, as well as the intraoral condition, such as tooth position on the arches.

Molars (M) are considered those with more doubtful prognosis. ${ }^{6}$ The presence of furcation defects, divergence of roots and dimensions of the furcation entrances; concavities on the root surfaces; cervical enamel 
projections; and even the more posterior position in the arches are considered some of the factors that influence the establishment and progression of periodontal destruction of those teeth. ${ }^{7}$ For example, increased periodontal probing depth (PPD), clinical attachment loss (CAL) and a higher percentage of missing teeth are observed for M..$^{8-11}$ In this scenario, the initial observations of Nordland et al. ${ }^{12}$ and Loos et al. ${ }^{13}$ are particularly important. They compared free surfaces of $\mathrm{M}$ and non-molars (NM) with those having furcation involvement and observed that the worst condition was always associated with M. Nevertheless, most studies have not evaluated the response of different teeth to systematic periodontal intervention. Prasad et al. ${ }^{14}$ observed that, regardless of the arch, gender or experiment period, $\mathrm{M}$ harbored a significantly higher frequency of plaque as compared to NM or to the whole arch values. Similar behavior was reported by Sreenivasan et al. ${ }^{15}$. They observed higher biofilm and gingivitis scores, as well as higher bacterial counts, in $\mathrm{M}$. Furthermore, de novo plaque formation may differ between the teeth groups. Furuichi et al. ${ }^{16}$ reported that $\mathrm{M}$, especially the mandibular ones, had higher plaque scores during a shorter time period.

Studies investigating the efficacy of various treatments to remove the subgingival biofilm also highlighted the possible effects of differences between the teeth with respect to their anatomy and positions in the arch. Starting with the results of Pihlstrom et al. ${ }^{8}$ in the 1980 s to those of Rosling et al. in 2001, ${ }^{10}$ it was observed, in patients undergoing maintenance, that M, compared with NM, showed higher PPDs and CAL means.

On the other hand, periodontal condition is also affected by risk factors, particularly tobacco and diabetes. Smokers have been reported to have the worst periodontal conditions. However, although smoking is clearly related to the establishment and progression of periodontitis, ${ }^{17}$ its association with local factors is still unknown.

So, the aim of the present secondary analysis was to determine to which extent $\mathrm{M}$ and NM teeth benefit from a strictly supragingival biofilm control in both smoker and never-smoker periodontal patients.

\section{Methodology Ethical considerations}

This investigation was conducted in accordance with the Declaration of Helsinki. The protocol was approved by the ethics committee of the Federal University of Rio Grande do Sul (UFRGS).

\section{Study design and sample}

This is a secondary analysis of a previous longitudinal, single-arm clinical trial with supragingival biofilm control as the intervention and smoking as a risk factor. ${ }^{4}$

\section{Sample}

Sample size was calculated based on PPD, wherein it was estimated that to achieve $80 \%$ power to detect differences in PPD of $0.8-1.0 \mathrm{~mm}$ among smokers (S) and never-smokers (NS) with $\alpha<0.05,25$ individuals in each experimental group were needed.

A consecutive sample was selected from those patients seeking periodontal treatment at the School of Dentistry of UFRGS (July 2003-August 2004)

The volunteers underwent a clinical exam if they initially met the following criteria:

- signing an informed consent;

- absence of an adverse systemic condition;

- non-pregnant woman;

- no periodontal treatment in the previous 12 months;

- no intake of antibiotic or anti-inflammatory in the past 6 and 3 months, respectively;

- absence of prescription to a regular chemical plaque control.

Eligible subjects were then included if they had at least 12 teeth (excluding third molars and endoperiodontally involved teeth). Moreover, each participant should present at least 4 sites with a PPD of $3-5 \mathrm{~mm}$ and 4 with $6-10 \mathrm{~mm}$, in different teeth with gingivitis and periodontitis. Fifty subjects with gingivitis and moderate-to-severe chronic periodontitis ${ }^{17}$ were included (Table 1). 
Table 1 - Baseline characteristics of patients according to experimental group.

\begin{tabular}{l|c|l}
\hline Variable & Never-smokers & Smokers \\
\hline $\mathrm{N}$ & 25 & 25 \\
\hline Age $^{*}$ & $46.8 \pm 7.1$ & $45.9 \pm 5.4$ \\
\hline Gender (\% males) & 40 & 56 \\
\hline Number of teeth* & $21.4 \pm 3.8$ & $21.2 \pm 3.7$ \\
\hline Cigarettes per day* $^{*}$ & $0 \pm 0$ & $19.4 \pm 11.6$ \\
\hline Years of smoking* & $0 \pm 0$ & $24.8 \pm 8.6$ \\
\hline
\end{tabular}

*mean \pm standard deviation.

\section{Reproducibility measures}

One trained and calibrated examiner (F.B.P.) reached a weighted Kappa value $( \pm 1 \mathrm{~mm})$ for PPD of 0.98 and 0.95 , respectively, before and during the study.

\section{Clinical Procedures}

Periodontal examination

A full-mouth exam (periodontal probe, Neumar Inc., São Paulo, Brazil) was conducted at six sites per tooth (mesiobuccal, buccal, distobuccal, mesiolingual, lingual, distolingual). Visible plaque (VPI $)^{18}$ and Gingival bleeding $(\mathrm{GBI})^{18}$ indexes, PPD, bleeding on probing (BOP) and CAL were assessed. PPD and CAL measurements were taken in millimeters and rounded to the nearest whole millimeter.

Clinical data were collected at day 0 (baseline), 30 and 180.

\section{Experimental interventions}

Strict supragingival control was established from days 0 to 180 (Figure 1). Initially the subjects received full-mouth supragingival debridement (Nos. 11-12; 13-14 Gracey curettes; Neumar) and oral hygiene instructions. Briefly, subjects were trained in the Bass technique. A soft-bristle toothbrush and a fluoride toothpaste (CloseUp, Unilever, Valinhos, Brazil) were provided along 180 days. ${ }^{4}$ The patients were supervised weekly, and their adherence to the clinical instructions, as well as to supragingival control (VPI and GBI), was checked. Reinforcement regarding oral hygiene was provided on an individual basis by a periodontist (MSc), not involved with the clinical exams (S.C.G).

\section{Statistical analysis}

Data analysis was performed using the computer program SPSS v.17.0 (SPSS Inc., Chicago, USA). Level of significance was set at $5 \%$, and the individual was considered as the unit of analysis.

The analysis included 50 individuals with NM (25 S; $25 \mathrm{NS}$ ) and 48 individuals with M (25 S; 23 NS) since two patients had no M. For the descriptive analysis, NM (incisors, canines and premolars) and $M$ (molars) teeth were grouped within an individual. In sequence, the average percentage of sites positive to VPI, GBI and BOP was calculated. Mean values were calculated for PPD and CAL. Normal distribution of data was tested by the Kolmogorov-Smirnov test. Linear models for two factors (smoking and dental group) were used to calculate estimates (mean $\pm \mathrm{SD}$ ) for comparison at baseline. Reductions in periodontal indicators at the end of the experimental period were also calculated and analyzed. The multilevel analysis considered sites, nested in teeth, and nested in patients, by means of linear mixed models.

\section{Results}

Baseline data are expressed in Table 2. It can be observed that VPI ( $\mathrm{p}=0.017)$, PPD $(\mathrm{p}<0.001)$ and CAL $(\mathrm{p}<0.001)$ were significantly higher in $\mathrm{M}$ than in NM. When exposure to tobacco was considered, smokers showed more pronounced CAL for both $\mathrm{M}$ and NM ( $\mathrm{p}=0.007)$.

The percentage reduction in periodontal indicators throughout the treatment is shown in Table 3. Significant and similar reductions were observed for all indicators during the study. The greatest reductions were observed within the first 30 days (data not shown). Smoking did not affect the response to therapy. However, the reductions observed in CAL for $\mathrm{M}$ (NS $0.57 \pm 0.50 ; S 0.67 \pm 0.64$ ) compared with NM (NS $0.38 \pm 0.23$; S $0.51 \pm 0.33$ ) were close to being statistically significant $(\mathrm{p}=0.05)$.

Multilevel analysis revealed that only for PPD reduction was the interaction between the three levels (sites, teeth and patient) significant $(\mathrm{p}<0.001)$. Reductions were higher for approximal surfaces (NS: $\mathrm{NM}=1.22 \pm 1.33 ; \mathrm{M}=1.76 \pm 1.81$; $\mathrm{S}: \mathrm{NM}=1.35 \pm 1.33 ; \mathrm{M}=1.37 \pm 1.57) \mathrm{com}-$ 
Figure 1 - Flowchart of experimental procedures.
25 never-smokers and 25 smokers diagnosed with moderate to severe, widespread, periodontitis $(n=50)$

- Periodontal examination (VPI, GBI, PD, BOP and PI);

- Strict supragingival control performed in all patients;

- Individualized oral hygiene instructions $(\mathrm{OHI})$.

Day 0

- Reinforce and re-evaluation (VPI and GBI) of oral hygiene practices.

Weekly

- Periodontal examination (VPI, GBI, PD, BOP and PI);

- Reinforce of oral hygiene practices.

Day 30

- Reinforce and re-evaluation (VPI and GBI) of oral hygiene practices.

Weekly

- Periodontal examination (VPI, GBI, PD, BOP and PI).

Day 180

pared with free surfaces (NS: NM $=0.62 \pm 1.10$, $\mathrm{M}=0.67 \pm 1.24 ; \mathrm{S}: \mathrm{NM}=0.71 \pm 1.08$, $\mathrm{M}=0.93 \pm 1.39$ ) (Table 4$)$.

\section{Discussion}

This investigation showed that both supra- and subgingival periodontal indicators were reduced for non-molar and molar teeth after a regimen of strict supragingival plaque control. Reductions observed for PPD were greater for M sites, while CAL did not differ between $\mathrm{M}$ and NM. Smoking did not influence this response.

The present study was a secondary analysis from a study reported earlier, performed as a single-arm clinical trial, ${ }^{4}$ that is, a single treatment distributed among different groups. Thus, randomization of patients or treatment was not performed. Furthermore, because of the longitudinal nature of the investigation and also to the intra- and extraoral characteristics related to tobacco use, it was not possible to ensure that the examiner remained blinded to the study group. However, along the study subjects were identified by number only and the examiner did not have access to the patients' general health records.

Previous studies ${ }^{4,5}$ have shown that moderateto-severe periodontitis patients benefited markedly from a supragingival control regimen, although an analysis of the differences between $\mathrm{M}$ and $\mathrm{NM}$ was not performed. The literature identifies $M$ teeth as those with poorer clinical status and prognosis. ${ }^{7,8,11}$ 
Table 2 - Baseline periodontal indicators for patients subjected to supragingival control.

\begin{tabular}{|c|c|c|c|c|}
\hline & & NM & $M$ & \\
\hline \multirow{3}{*}{$\begin{array}{l}\text { VPI } \\
(\%)^{*}\end{array}$} & NS & $89.9 \pm 08.9$ & $95.7 \pm 06.8$ & \multirow{2}{*}{$p=0.175$} \\
\hline & $S$ & $87.1 \pm 14.6$ & $92.3 \pm 12.4$ & \\
\hline & \multicolumn{4}{|c|}{$p=0.017$} \\
\hline \multirow{3}{*}{$\begin{array}{l}\text { GBI } \\
(\%)^{*}\end{array}$} & NS & $82.5 \pm 13.7$ & $83.5 \pm 20.3$ & \multirow{2}{*}{$p=0.064$} \\
\hline & $S$ & $74.7 \pm 19.6$ & $77.4 \pm 19.2$ & \\
\hline & \multicolumn{4}{|c|}{$p=0.621$} \\
\hline \multirow{3}{*}{$\begin{array}{l}\text { BOP } \\
(\%)^{*}\end{array}$} & NS & $14.3 \pm 10.5$ & $17.7 \pm 18.6$ & \multirow{2}{*}{$p=0.109$} \\
\hline & $S$ & $22.1 \pm 16.4$ & $21.5 \pm 22.9$ & \\
\hline & \multicolumn{4}{|c|}{$p=0.688$} \\
\hline \multirow{3}{*}{$\begin{array}{c}\text { PPD } \\
(\mathrm{mm})^{*}\end{array}$} & NS & $3.49 \pm 0.50$ & $4.54 \pm 0.91$ & \multirow{2}{*}{$p=0.356$} \\
\hline & $S$ & $3.78 \pm 0.65$ & $4.53 \pm 0.75$ & \\
\hline & \multicolumn{4}{|c|}{$p=0.000$} \\
\hline \multirow{3}{*}{$\begin{array}{c}\mathrm{CAL} \\
(\mathrm{mm})^{*}\end{array}$} & NS & $3.23 \pm 0.94$ & $4.39 \pm 1.38$ & \multirow{2}{*}{$p=0.007$} \\
\hline & $S$ & $4.05 \pm 1.12$ & $4.88 \pm 1.23$ & \\
\hline & \multicolumn{4}{|c|}{$p=0.000$} \\
\hline
\end{tabular}

NS: never-smokers; S: smokers; NM: non-molar; M: molar; VPI: visible plaque index; $\mathrm{GBI}$ : gingival bleeding index; $\mathrm{BOP}$ : bleeding on probing; PPD: periodontal probing depth; CAL: clinical attachment loss. *mean \pm standard deviation. Bold letters: Comparison between $M$ and NM. Italic letters: Comparison between S and NS.
Table 3 - Mean reduction in periodontal indicators at the end of the experimental period.

\begin{tabular}{|c|c|c|c|c|}
\hline & & NM & M & \\
\hline \multirow{3}{*}{$\begin{array}{l}\text { VPI } \\
(\%)^{* \pi}\end{array}$} & NS & $82.9 \pm 10.7$ & $79.9 \pm 16.0$ & \multirow[t]{2}{*}{$p=0.772$} \\
\hline & $S$ & $82.4 \pm 13.7$ & $78.6 \pm 19.4$ & \\
\hline & \multicolumn{4}{|c|}{$p=0.266$} \\
\hline \multirow{3}{*}{$\begin{array}{c}\text { GBI } \\
(\%)^{* \pi}\end{array}$} & NS & $80.2 \pm 12.7$ & $82.0 \pm 19.6$ & \multirow[t]{2}{*}{$p=0.131$} \\
\hline & $S$ & $74.4 \pm 19.6$ & $76.6 \pm 18.7$ & \\
\hline & \multicolumn{4}{|c|}{$p=0.581$} \\
\hline \multirow{3}{*}{$\begin{array}{l}\text { BOP } \\
(\%)^{* \pi}\end{array}$} & NS & $74.5 \pm 13.7$ & $65.6 \pm 19.1$ & \multirow[t]{2}{*}{$p=0.782$} \\
\hline & $S$ & $70.8 \pm 19.8$ & $66.9 \pm 29.6$ & \\
\hline & \multicolumn{4}{|c|}{$p=0.142$} \\
\hline \multirow{3}{*}{$\begin{array}{c}\text { PPD } \\
(\mathrm{mm})^{* \pi}\end{array}$} & NS & $1.01 \pm 0.41$ & $1.36 \pm 0.75$ & \multirow[t]{2}{*}{$p=0.963$} \\
\hline & $S$ & $1.13 \pm 0.53$ & $1.25 \pm 0.66$ & \\
\hline & \multicolumn{4}{|c|}{$p=0.059$} \\
\hline \multirow{3}{*}{$\begin{array}{c}\mathrm{CAL} \\
(\mathrm{mm})^{* \pi}\end{array}$} & NS & $0.38 \pm 0.23$ & $0.57 \pm 0.50$ & \multirow[t]{2}{*}{$p=0.226$} \\
\hline & $S$ & $0.50 \pm 0.33$ & $0.67 \pm 0.64$ & \\
\hline & \multicolumn{4}{|c|}{$p=0.050$} \\
\hline
\end{tabular}

NS: never-smokers; S: smokers; NM: non-molar; M: molar; VPI: visible plaque index; $\mathrm{GBI}$ : gingival bleeding index; $\mathrm{BOP}$ : bleeding on probing; PPD: periodontal probing depth; CAL: clinical attachment loss. *mean \pm standard deviation. ${ }^{\pi}$ Significant reduction for all indicators: $p=0.000$. Bold letters: Comparison between M and NM. Italic letters: Comparison between S and NS.

Table 4 - Multilevel analysis of mean reduction in periodontal indicators at the end of the experimental period.

\begin{tabular}{|c|c|c|c|c|c|c|c|}
\hline & & & VPI (\%)* & GBI (\%)* & $\mathrm{BOP}(\%)^{*}$ & $\mathrm{PPD}(\mathrm{mm})^{*}$ & $\mathrm{CAL}(\mathrm{mm})^{*}$ \\
\hline \multirow{4}{*}{ NS } & \multirow{2}{*}{ NM } & $\mathrm{FS}(\mathrm{n}=877)$ & $77.2 \pm 42.5$ & $63.3 \pm 49.1$ & $74.9 \pm 43.6$ & $0.62 \pm 1.10$ & $0.15 \pm 1.07$ \\
\hline & & AS $(n=1754)$ & $85.5 \pm 35.7$ & $88.7 \pm 31.6$ & $74.7 \pm 43.7$ & $1.22 \pm 1.33$ & $0.52 \pm 1.22$ \\
\hline & \multirow{2}{*}{ M } & $\mathrm{FS}(\mathrm{n}=192)$ & $84.4 \pm 36.4$ & $76.6 \pm 42.4$ & $72.4 \pm 45.9$ & $0.67 \pm 1.24$ & $0.36 \pm 1.29$ \\
\hline & & $\mathrm{AS}(\mathrm{n}=383)$ & $78.9 \pm 40.8$ & $90.1 \pm 29.9$ & $63.7 \pm 48.1$ & $1.76 \pm 1.81$ & $0.68 \pm 1.55$ \\
\hline \multirow{4}{*}{$S$} & \multirow{2}{*}{ NM } & $\mathrm{FS}(\mathrm{n}=860)$ & $74.1 \pm 44.3$ & $55.1 \pm 49.7$ & $70.9 \pm 45.6$ & $0.71 \pm 1.08$ & $0.28 \pm 1.10$ \\
\hline & & $\mathrm{AS}(\mathrm{n}=1720)$ & $87.0 \pm 33.8$ & $85.1 \pm 35.6$ & $69.9 \pm 46.6$ & $1.35 \pm 1.33$ & $0.61 \pm 1.26$ \\
\hline & \multirow{2}{*}{ M } & $F S(n=200)$ & $76.5 \pm 43.6$ & $61.0 \pm 48.8$ & $67.5 \pm 46.9$ & $0.93 \pm 1.39$ & $0.50 \pm 1.39$ \\
\hline & & $\mathrm{AS}(\mathrm{n}=400)$ & $81.5 \pm 38.8$ & $86.0 \pm 34.7$ & $62.2 \pm 49.5$ & $1.37 \pm 1.57$ & $0.67 \pm 1.56$ \\
\hline \multicolumn{2}{|c|}{$P$ value } & & 0.262 & 0.199 & 0.496 & 0.000 & 0.510 \\
\hline
\end{tabular}

NS: never-smokers; S: smokers; NM: non-molar; M: molar; FS: free sites; AS: approximal sites; VPI: visible plaque index; GBI: gingival bleeding index; BOP: bleeding on probing; PPD: periodontal probing depth; CAL: clinical attachment loss. ${ }^{*}$ mean \pm standard deviation.

This association may have been determined by anatomic features and by their positions in the arches, which supposedly influence biofilm control during daily hygiene procedures. ${ }^{7}$ The latter could influence the subgingival environment and thus long-term periodontal stability. ${ }^{3}$

In this study, it was observed that the $\mathrm{M}$ showed significantly higher values of VPI than did NM at 
baseline. Sreenivasan et al. ${ }^{15}$ reported higher plaque scores with de novo plaque formation being more pronounced in $\mathrm{M}$. The present analysis showed that the percentage of visible biofilm declined significantly during the first 30 days for $\mathrm{M}$ and $\mathrm{NM}$ and remained the same between groups throughout the study (data not shown). Tobacco exposure did not affect the response.

On the other hand, the GBI was not affected by tooth type $(\mathrm{p}=0.621)$ or by smoking $(\mathrm{p}=0.064)$. During the experimental period, even though differences were observed between $\mathrm{S}$ and NS in the 30-to-80-day interval (data not shown), the reduction achieved at day 180, compared with baseline, showed no differences. To the best of our knowledge, the relation between smoking and tooth type had never before been investigated. However, Sreenivasan et al..$^{15}$ found more gingival inflammation in $\mathrm{M}$ than in NM.

The present investigation also demonstrated that, at baseline, PPD values were significantly greater in $\mathrm{M}(4.54 \pm 0.91 ; 4.53 \pm 0.75)$ than in $\mathrm{NM}$ $(3.49 \pm 0.50 ; 3.78 \pm 0.65)$, respectively, for NS and S. Interestingly smoking did not influence these results. Our initial PPD values are higher than those observed by Rosling et al., ${ }^{10}$ who studied normal (NM $2.5 \pm 0.4 ;$ M $2.8 \pm 0.5$ ) and highly susceptible (NM $2.9 \pm 0.6 ;$ M $4.0 \pm 0.8)$ individuals. This author, as reported by the present study, also observed higher and significant PPD values for M. Miyamoto et al. ${ }^{11}$ likewise reported similar results for $\mathrm{NM}$ $(3.50 \mathrm{~mm})$ and $\mathrm{M}(4.36 \mathrm{~mm})$.

It was also observed that CAL values were higher for $\mathrm{M}$ at baseline. Pihlstrom et al. ${ }^{8}$ observed that $\mathrm{M}$ had greater CAL (M $4.14 \mathrm{~mm}$ and NM $3.40 \mathrm{~mm}$, with a difference of $0.74 \pm 0.1 \mathrm{~mm}$ between them). The reported data are similar to ours: M (NS $4.39 \pm 1.38 ; S 4.88 \pm 1.23$ ) and NM (NS $3.23 \pm 0.94$; $S 4.05 \pm 1.12$ ).

Since there were differences between $\mathrm{M}$ and NM at baseline, an analysis solely expressed by means would not identify eventual differences in response to the supragingival control throughout the experimental period. Thus, the mean percentage of reduction, for both supra- and subgingival indicators, was calculated. In general, it was observed that $\mathrm{M}$ and
NM responded similarly to treatment, except for the clinical attachment gain that was greater for $\mathrm{M}$. However, it must be realized that, even with numerical differences for the mean reduction among the groups, the significance had a $\mathrm{p}$ value at the limit $(\mathrm{p}=0.05)$ that underscores the importance of discussing the clinical implications of such finding.

Our data showed that smoking did not influence the response to therapy, which is in accordance with other studies. Some authors showed that, although S may exhibit a worse periodontal clinical condition, their response to periodontal therapy is somewhat similar to that obtained in NS. ${ }^{4,19}$

Finally, considering the interdependence between tooth sites, teeth and patients, ${ }^{20}$ a multilevel analysis was performed using all six tooth sites. In sequence, the analysis took into consideration the approximal and free surfaces as a whole. Interestingly, PPD reductions were greater at the approximal sites, in molar teeth, in smoker patients (Table 4). As this result did not differ from those obtained on all sites individually, these data are not shown. This finding seems to confirm the trend observed for PPD values expressed as means grouped by individuals $(\mathrm{p}=0.059)$ (Table 3$)$ and furthermore strengthens the effect of supragingival control even in areas having difficult access.

It is important to emphasize that, since the present study is a secondary analysis, a sample size was not calculated specifically to compare M and NM. However, because significant differences could be demonstrated, the number of individuals appears to be adequate. Nevertheless, to strengthen and confirm the present results, clinical trials specifically designed to address whether $\mathrm{M}$ and $\mathrm{NM}$ respond similarly to periodontal treatment are needed.

\section{Conclusions}

Once adequate supragingival control is established, even teeth having a poor prognosis are benefited, irrespective of tobacco exposure, from the standpoint of both inflammation and clinical attachment gain. Thus, the supragingival condition should be considered in determining the prognosis of molar teeth, regardless of the presence of known risk factors. 


\section{Acknowledgements}

National Research Council, Brasília, DF, Brazil (140428/2003-8); National Coordination of Post-

\section{References}

1. Axelsson P, Nyström B, Lindhe J. The long-term effect of a plaque control program on tooth mortality, caries and periodontal disease in adults. Results after 30 years of maintenance. J Clin Periodontol. 2004 Sep;31(9):749-57.

2. Tezal M, Scannapieco FA, Wactawski-Wende J, Grossi SG, Genco RJ. Supragingival plaque may modify the effects of subgingival bacteria on attachment loss. J Periodontol. 2006 May;77(5):808-13.

3. Weidlich P, Souza MAL, Oppermann RV. Evaluation of the dentogingival area during early plaque formation. J Periodontol. 2001 Jul;72(7):901-10.

4. Gomes SC, Piccinin FB, Susin C, Oppermann RV, Marcantonio RAC. Effect of supragingival plaque control in smokers and neversmokers: 6-month evaluation of patients with periodontitis. J Periodontol. 2007 Aug;78(8):1515-21.

5. Gomes SC, Piccinin FB, Oppermann RV, Susin C, Marcantonio RA. The effect of smoking on gingival crevicular fluid volume during the treatment of gingivitis. Acta Odontol Latinoam. 2009;22(3):201-6.

6. Heitz-Mayfield LJA, Trombelli L, Heitz F, Needleman I, Moles D. A systematic review of the effect of surgical debridement vs. non-surgical debridement for the treatment of chronic periodontitis. J Clin Periodontol. 2002;29 Suppl 3:92-102.

7. Matthews DC, Tabesh M. Detection of localized tooth-related factors that predispose to periodontal infections. Periodontol 2000. 2004;34:136-50.

8. Pihlstrom BL, Oliphant TH, McHught RB. Molar and nonmolar teeth compared over $61 / 2$ years following two methods of periodontal therapy. J Periodontol. 1984 Sep;55(9):499-504.

9. Svardström G, Weenström JL. Periodontal treatment decisions for molars: an analysis of influencing factors and long-term outcome. J Periodontol. 2000 Apr;71(4):579-85.

10. Rosling B, Serino G, Hellström MK, Socransky SS, Lindhe J. Longitudinal periodontal tissue alterations during supportive therapy. Findings from subjects with normal and high sus-
Graduate Education, Brasília, DF, Brazil (0550/043); and São Paulo Research Foundation, SP, Brazil (2003/09302-8).

ceptibility to periodontal disease. J Clin Periodontol. 2001 Mar;28(3):241-9.

11. Miyamoto T, Kumagai T, Lang MS, Nunn ME. Compliance as a prognostic indicator. II. Impact of patient's compliance to the individual tooth survival. J Periodontol. 2010 Sep;81(9):12808.

12. Nordland P, Garrett S, Kiger R, Vanooteghem R, Hutchens $\mathrm{LH}$, Egelberg J. The effect of plaque control and root debridement in molar teeth. J Clin Periodontol. 1987 Apr;14(4):231-6.

13. Loos B, Nylund K, Claffey N, Egelberg J. Clinical effects of root debridement in molar and non-molar teeth. A 2-year follow-up. J Clin Periodontol. 1989 Sep;16(8):498-504.

14. Prasad KV, Sreenivasan PK, Patil S, Chhabra KG, Javali SB, DeVizio W. Removal of dental plaque from different regions of the mouth after a 1-minute episode of mechanical oral hygiene. Am J Dent. 2011 Feb;24(1):60-4.

15. Sreenivasan PK, DeVizio W, Prasad KV, Patil S, Chhabra KG, Rajesh G, et al. Regional differences within the dentition for plaque, gingivitis, and anaerobic bacteria. J Clin Dent. 2010;21(1):13-9.

16. Furuichi Y, Lindhe J, Ramberg P, Volpe AR. Patterns of de novo plaque formation in the human dentition. J Clin Periodontol. 1992 Jul;19(6):423-33.

17. Armitage GC. Development of a classification system for periodontal diseases and conditions. Ann Periodontol. 1999 Dec;4(1):1-6

18. Ainamo J, Bay I. Problems and proposals for recording gingivitis and plaque. Int Dent J. 1975 Dec;25(4):229-35.

19. Darby IB, Hodge PJ, Riggio MP, Kinane DF. Clinical and microbiological effect of scaling and root planning in smoker and non-smoker chronic and aggressive periodontitis patients. J Clin Periodontol. 2005 Feb;32(2):200-6.

20. Albandar JM, Goldstein H. Multi-level statistical models in studies of periodontal diseases. J Periodontol. 1992 Aug;63(8):690-5. 